\title{
Design of Embedded Environment Monitoring System based on Nios II
}

\author{
Wanxu Zhang ${ }^{1, a}$, Tingting $\mathrm{Xia}^{2, \mathrm{~b}}$ and Bo Jiang ${ }^{3, \mathrm{c}}$ \\ 1,2,3 School of Information Science and Technology,Northwest University,Xi'an 710127, China \\ awxzhang@nwu.edu.cn, bxtt_128@163.com, cjiangbo@nwu.edu.cn
}

Keywords: Nios II, $\mu$ CLinux, CGI, AJAX

\begin{abstract}
An embedded environment monitoring system that can be applied in warehouses, museums, families and other occasions is designed and implemented in this paper by using high-performance Nios II and open source embedded operating system. The system realizes the data display, data analysis, parameter settings, user information management, system settings and other functions with the advantages of stability, accuracy, reliability and convenience. And it effectively reduces the burden of the server and achieves an asynchronous dynamic interactive web through AJAX technology and CGI.
\end{abstract}

\section{Introduction}

With the rapid development of embedded technology and the widespread popularity of Internet, embedded devices accessing the network are becoming increasingly popular. The communication between embedded devices and other devices in the network can be implemented with the integration of embedded and Internet technology, which facilitates remote monitoring. Therefore, authorized users can access remote embedded device for monitoring and management anywhere[1].

Due to a scalable, customizable Nios II processor as the core hardware, and an embedded $\mu$ CLinux system as the software platform, this system uses the embedded web server (boa) to build on the software and hardware platform and CGI programs running on the boa to store, analyze and manage the information collected by the nodes in the WSN. Users can remotely access and manage embedded devices through a web browser, and ultimately achieves data analysis, parameter settings, user information management, system settings, and other functions.

\section{System Design}

Embedded environment monitoring system includes hardware circuit, operating system, network communications and applications.

Hardware circuit. The FPGA chip (EP1C12Q240C8N) supporting Nios II processor acquires the most appropriate processor, peripherals and interface combinations by customizing Nios II soft-core, to achieve a stable, efficient data storage, analysis and management capabilities.

Operating system. It uses the proprietary environmental monitoring oriented operating system, to achieve data storage and remote transmission. Based on good $\mu$ Clinux's stability and portability, powerful networking capabilities, excellent file system, the rich standard API as well as the support of TCP / IP network protocol, it not only realizes functions of the system but also improves the performance of equipment through $\mu$ Clinux cutting.

Network communication. The system needs Common Internet File System (CIFS) to achieve file sharing between $\mu$ CLinux and Windows. Samba is the most common way to achieve CIFS protocol on the $\mu$ Clinux system, so it is adopted .

Applications. The system achieves data display, data analysis, parameter settings, user information management, system settings and other functions by CGI programs, which enable users to remotely access and manage embedded devices via a Web browser. 


\section{Hardware Design}

Hardware includes FPGA, storage devices, ethernet controller and interface. Block diagram of hardware is shown in Fig. 1.

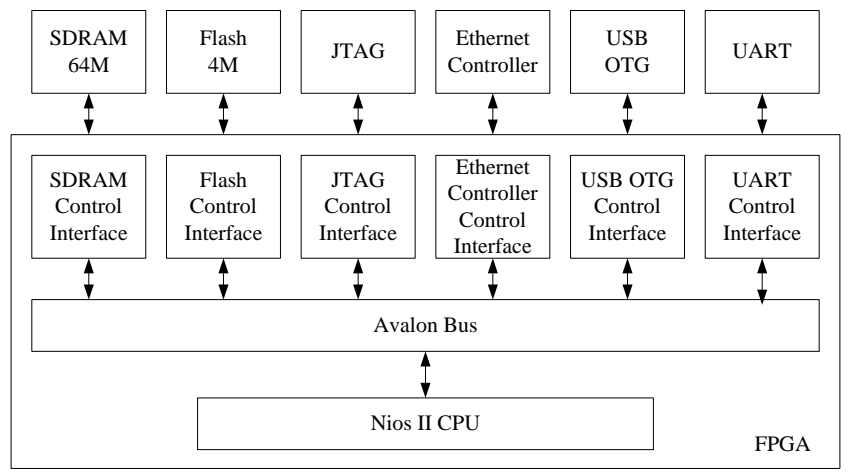

Fig. 1 Block diagram of hardware

The functions of key components are described as follows:

SDRAM: capacity of 64M, provises the workspace of systems program. System data, user data and stack are located in SDRAM memory.

Flash: capacity of 4M, used to store user applications, embedded Linux operating system and other user data needed to be saved after system power-down.

USB OTG: interface of U disk or hard disk and other storage devices used to store user information and monitoring data.

Ethernet controller: provides a physical channel used to access Ethernet. Ethernet controller uses DM9000AE device, which has a 10M / 100M physical transceiver interface and a general processor interface. We complete Interface customization according to the SOPC Builder design wizard when designing the user interface.

JTAG: interface for system debugging and programming .

UART: interface for data communication with the sink node in wireless sensor network.

Nios II Soft-core Processor Customization. Nios II soft-core processor is a 32-bit RISC processor developed to support FPGA products by ALTERA company[2]. The system uses a Nios II/f soft-core and 32-bit 100MHZ CPU to achieve connectivity between processor and SDRAM, Flash, RTC, USB OTG, UART, JTAG and Ethernet control interfaces, through the Avalon Bus.

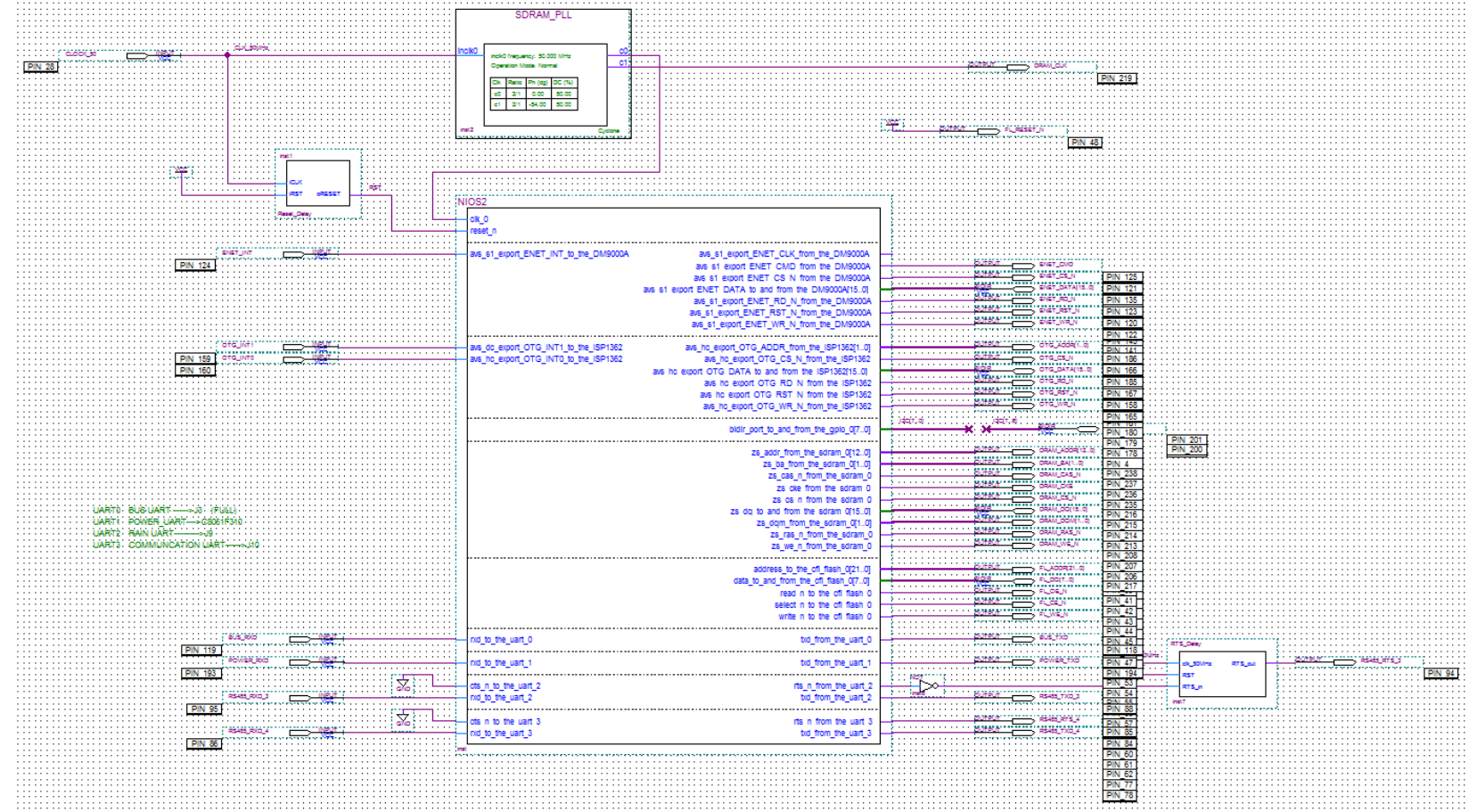

Fig. 2 Design of the top-level hardware module 
The Design of the top-level Hardware Module. The design of the top-level hardware module is shown in Fig. 2. This module is composed of PLL and NiosII soft core processor, among which the output clock frequency of PLL is $100 \mathrm{MHz}$ and it is used to set the SDRAM. After completing the design of the top-level hardware module, we compile and run the system to generate the configuration file nios2.ptf.

\section{Design of the Software System}

In this paper, the design of the software system uses B/S Mode. With the embedded web server building on the hardware platform, users can directly send a request to the server via the browser and access environmental data, so as to achieve the environmental monitoring[3].The specific design and implementation of the software system are discussed as follows.

Migration of Operating System. $\mu$ Clinux is the good open-source embedded operating system suitable for the hardware platform without MMU. Its core is small and its functions are powerful, which contributes to the task scheduling of monitoring equipment. Cross-development environment is a PC that runs an Ubuntu 12.04 operating system, and cross-compilation tool is nios2gcc. After configuring the kernel and loading profiles nios2.ptf, we can compile and build the system image zImage. The migration of the operating system $\mu$ Clinux can be completed through downloading the zImage to the target board.

Duilding Boa Server. Boa is a small and single-task http web server characterized by open source code, excellent performance and support for CGI programs, especially suitable for the application in embedded system[4]. The building of boa server can be completed by the following steps: Modify the configuration of boa by boa.conf file(storage directory of index.html and CGI programs etc); Select boa when configuring the kernel(uClinux Distribution Configuration --->Network Applications --->boa); Compile successfully.

The Design of Application Management Program. Users interact with the Web server through a browser, but usually HTML pages only display static data. Therefore, dynamic web technology for this application on the web server is necessary[5].Currently, there are four kinds of common dynamic web pages techniques:CGI 、ASP 、 PHP and JSP. Since CGI is a good cross-platform and programming language independence technology, it is used in this system.

We can enter the main page index.html by entering the IP address(http://192.168.10.250/) of embedded environmental monitoring system in the browser. To ensure the security of the system, users need to be authenticated before entering the environmental monitoring system.

<form method="post" action="http://192.168.10.250/cgi-bin/login.cgi">

The login.cgi program calls are embedded in the index.html through the "form", and passes user information to login.cgi by post method. The login.cgi compares the user information submitted by the form with user information stored in the database, and users can enter the environmental monitoring system homepage after successful authentication.

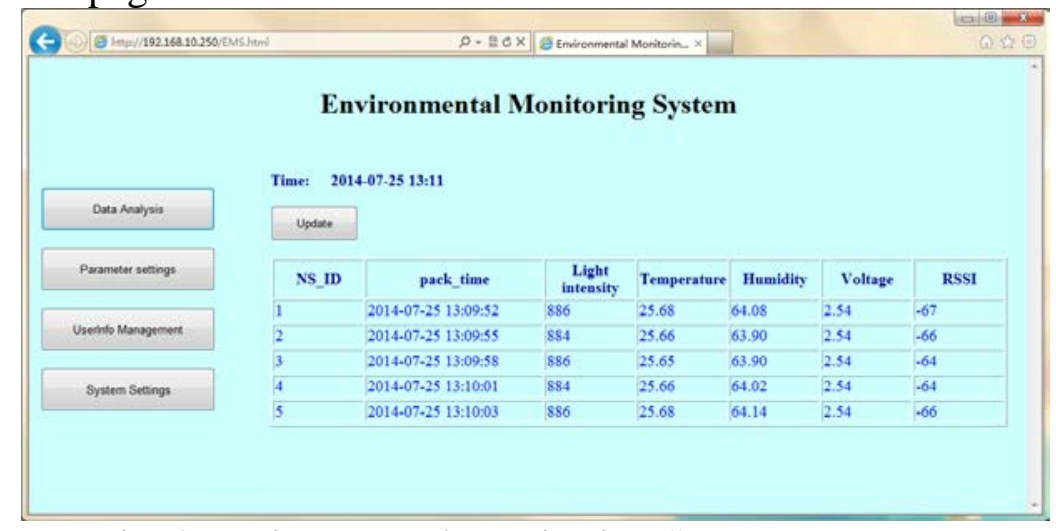

Fig. 3 Environmental Monitoring System Homepage

Environmental monitoring systems homepage includes 5 sections: data display, data analysis, parameter settings, user information management and system settings. Environmental monitoring 
system homepage mainly displays the current environmental monitoring status, namely, data display section, and provides buttons to switch to the other four sections. Data analysis performs statistic of related data information; Parameter settings set related data parameters, such as alarm threshold settings, etc; User information management can modify, delete user information and so on. System settings include permissions settings, network settings, etc.

In this system, environmental data is dynamicly updated by using AJAX technology[6]. Users can exchange the data with the server and update part of the page without loading the entire page. Part of the code is as follows:

varxmlhttp;

xmlhttp.onreadystatechange $=$ function()

$\{$ if (xmlhttp.readyState $==4$ \&\&xmlhttp.status $==200$ )

\{ document.getElementById("show_data").innerHTML=xmlhttp.responseText; \}

xmlhttp.open("GET","http://192.168.10.250/cgi-bin/show.cgi?t="+ Math.random(),true);

xmlhttp.send();

In this code, show.cgi is responsible for database query, then HTML statement is used to return the corresponding results.

Data analysis, parameter settings, user information management and system settings section can be switched by clicking on corresponding buttons. HTML code used to switch to the data analysis is as follows:

$<$ input type=|"submit $\mid " \quad$ value=|"Data Analysis $\backslash " \quad$ onclick=|"document.opt.action= 'http://192.168.10.250 /cgi-bin/data_a.cgi'|">

The data_a.cgi is CGI program of the data analysis part, in which the printf () functionis used to generate the corresponding HTML form, so do other parts.

\section{Conclusion}

An embedded environment monitoring system designed and implemented by using high-performance Nios II and open source embedded operating system can be applied in warehouses, museums, families and other occasions. It effectively reduces the burden of the server by AJAX technology and CGI to achieve an asynchronous dynamic interactive web.This design has such advantages as small size, low power, low cost, portable customizable software and hardware.

\section{References}

[1]HaoSulan, QiaoGangzhu, and Tan Ying. EnvironmentTemperature Monitoring System Based on Wireless Sensor Network[J].ElectronicSci.\&Tech. 2014,27(8):26-28.

[2] Zhang Rong, Huang Haiying, and Li Chunzhi.Software and Hardware Design of the Embedded Test System Based on NIOS II Soft-core Processor[J]. Computer Measurement \& Control. 2012,20(2):303-306.

[3] Zhao Jianhua, and ShiZhenwei.Realization of Embedded Web Server in Smart Home Control System[J].Computer Technology and Development,2013,23(3):164-167.

[4] Liang Yong'en, Wan Shiming,and Tang Hailin. Embedded Web Server in Meteorological Environment Monitoring System[J]. Computer Systems \& Applications, 2014(8):237-241.

[5]XuShuping, Li Jun, and Zhang Jun. Design and Implementation of Embedded Web Server Software[J]. Journal of Xi'an Technological University.2014,34(3):193-198.

[6] Chen Kansong, Liu Hongbo, and Xiong Pan.Implementation of dynamic displaying data of embedded Web server based on ARM9[J]. Internet of Things Technologies.2014, (3):18-23. 